\title{
Group transformation: fruiting body and stalk formation
}

\author{
Cathleen Broersma ${ }^{12}$, Elizabeth Ostrowski ${ }^{1}$ \\ ${ }^{1}$ School of Natural and Computational Sciences, Massey University, Auckland, New Zealand \\ ${ }^{2}$ Corresponding Author: c.broersma@massey.ac.nz
}

\begin{abstract}
Throughout the eukaryotic tree of life, amoeboid organisms have evolved that aggregate upon starvation and form multicellular fruiting bodies, consisting of a ball of spores atop a stalk. This chapter discusses the remarkable convergent evolution of a stalked fruiting body in these different taxa. It then discusses a well-studied group of aggregative fruiters, the cellular slime molds, in more detail. These organisms exhibit substantial variation in their stalk formation and composition, which allows a better understanding of the evolution, maintenance and possible functions of stalked fruiting bodies, but also points to potential costs and benefits of different types of stalks.
\end{abstract}

Keywords: aggregative multicellularity, fruiting body, stalk formation, social amoebae, dictyostelium 
Dispersal is essential to the life-history of many organisms (Bowler and Benton 2005; Kokko and López-Sepulcre 2006). It can enhance survival when environmental conditions deteriorate, promote outbreeding, and broaden a species' geographic range. Dispersal often occurs in response to environmental cues, is regulated during ontogeny, and may involve specialised structures or cells (propagules) that promote long-distance travel and survival under adverse conditions.

Dispersal can potentially be accomplished by locomotion, and many single-celled organisms have mechanisms of active locomotion that enable travel over short distances. For example, some prokaryotic and eukaryotic microorganisms can swim using their flagella. Amoebae crawl across surfaces by extending and retracting pseudopods. Some organisms, such as myxobacteria, can glide over surfaces alone or in groups. However, microscopic organisms typically rely on passive mechanisms for dispersal over greater distances. Passive mechanisms include dispersal by wind or water or by hitching a ride on a larger organism. It can be facilitated by a resting state, such a spore or cyst.

In addition to forming hardy cysts or spores that protect cells in harsh environments, many organisms also produce fruiting bodies that promote dispersal by lifting spores up into the air. Fruiting bodies are produced by diverse taxa, ranging from fungi to bacteria to amoebae. Most have a similar morphology, consisting of some sort of stalk that lifts and supports a spore-producing head, resulting in a lollipop or umbrella morphology. Fruiting bodies can consist of only one or a few spores produced by cell division ("sporocarps") or they can be multicellular, usually produced through aggregation of cells ("sorocarps") (Spiegel et al. 2017; Kang et al. 2017). Formation of single-celled vs multicellular fruiting bodies likely entails different costs and benefits, as do the different ways of achieving these structures, through aggregation or cell division, referred to as "coming together" versus "staying together" (Tarnita, Taubes, and Nowak 2013). 
In this chapter, we discuss what is known about the evolution and function of stalked fruiting bodies in taxa that exhibit aggregative multicellularity. We begin by discussing amoeboid organisms throughout the tree of life that form these structures. The convergent evolution of similar morphologies, accomplished through diverse means, suggests that they are adaptations. Nevertheless, while it seems likely that stalked fruiting bodies confer a benefit, exactly what they are an adaptation for, and why they evolved and persist, remains subject to debate. In the later sections, we turn our attention to a large clade of sorocarpic amoebae-the Dictyostelia —-that are well-studied for their aggregative multicellularity from both a developmental and an evolutionary perspective.

\section{Aggregative fruiting is found throughout the eukaryotic tree of life}

Many taxa that undergo aggregative multicellularity to form fruiting bodies have an amoeboid single cell state. Thus, these organisms are called "sorocarpic amoebae" or "cellular slime molds". The first sorocarpic amoeba to be discovered, Dictyostelium mucoroides, was isolated by Brefeld in 1869 (Brefeld 1869). The Dictyostelia, which comprises a large clade within the Amoebozoa, is still the most well-studied of the taxa that undergo this morphological transformation (Shadwick et al. 2009). However, sorocarpic amoebae can be found in five of the six eukaryotic supergroups: Amoebozoa, Opisthokonta, Excavata, Stramenopiles and Rhizaria (Fig. 1). Molecular reconstruction indicates that sorocarpy evolved independently in most of these taxa, as summarized in Brown and Silberman (2013).

Sorocarpic amoebae have a characteristic life cycle, consisting of separate unicellular and multicellular stages, the latter of which is achieved through aggregation. In all cases, the transition to multicellularity involves a switch from a stage of feeding and cell division to one of development and differentiation. When nutrients are abundant, the single-celled amoebae feed on soil microorganisms and increase in number by cell division. Upon starvation, 
anywhere from a few to hundreds of thousands of amoebae aggregate through chemotaxis and cooperatively form a fruiting body structure, consisting of dispersal propagules and a stalk that supports them. Despite these commonalities, however, there are numerous differences among different types of sorocarpic amoebae in the formation and morphology of their fruiting bodies. Below, we emphasize some notable differences among the different taxa and how these different structures, achieved through different routes, nevertheless result in a stalked fruiting body structure (Fig. 1).

\section{Guttulinopsidae (Rhizaria)}

Species from the genus Guttulinopsis are the only known aggregative fruiters in the supergroup Rhizaria, which contains mostly unicellular eukaryotes (Brown et al. 2012). In the most common species, Guttulinopsis vulgaris, the stalk is composed of multiple different compartments, some of which contain dead cells, which can hold aloft one or several sori (Fig. 1A) (Raper, Worley, and Kessler 1977). Guttulinopsis thus represents an example of reproductive division of labor; only some cells undertake reproduction and others instead provide non-reproductive, structural support.

\section{Acrasidae (Excavata)}

The species Acrasis rosea was the first non-dictyostelid sorocarpic amoeba to be discovered (Lindsay S. Olive and Stoianovitch 1960). Following aggregation into a mound, a single cell at the base of the mound differentiates into an encysted stalk cell. Cells on top of the stalk cell subsequently encyst as well, resulting in a stalk that extends upward and holds aloft the rest of the population. After the stalk is complete, the remaining cells in the aggregate form chains and encyst to become spore cells. This results in a fruiting body that resembles a tree-like structure, consisting of a main branch and smaller offshoots (Fig. 1B). Although all cells in the fruiting body are viable, it is unknown whether there are fitness costs or benefits associated with adopting different positions in the fruiting body (Kaushik and Nanjundiah 2003). 


\section{Copromyxa (Amoebozoa)}

The genus Copromyxa consists of only two species, only one of which, Copromyxa protea, exhibits aggregative fruiting (Brown, Silberman, and Spiegel 2011). In Copromyxa, fruiting body formation involves aggregation of cells, with those at the apex becoming encysted (Fig 1C). Similar to A. rosea, the fruiting body consists of a column of cells, and the existence of any position-dependent fitness difference is unknown. Kaushik and Nanjundiah (2003) describe this process as "coming together and sticking to each other", a more primitive evolutionary form of multicellularity compared to other species of sorocarpic amoebae that show complex division of labor, where cells adopt distinct and irreversible cell fates.

\section{Fonticula (Opisthokonta)}

Multicellularity evolved multiple times within the Opisthokonta, manifested by different forms: metazoan (animals) and fungi, which arise by cell division from a single starting cell, and aggregative fruiting forms (Brown, Spiegel, and Silberman 2009; Ruiz-Trillo et al. 2007; Fisher, Shik, and Boomsma 2020). Among the latter is the taxon Fonticula, which currently consists of only a single species, Fonticula alba. F. alba was isolated only once by Olive and Stoianovitch (1960) and never rediscovered, although the original isolate has been retained. Following aggregation into a mound, the cells secrete a Golgi-derived extracellular matrix, forming a hollow volcano-shaped stalk tube. With the exception of a small number of cells that continue to produce stalk material, the cells in the stalk are mechanically forced out of the top of the structure. These cells encyst, forming a ball of spores suspended in a thin slime sheet, which collapses a few days after maturation (Fig. 1D) (Brown, Spiegel, and Silberman 2009; Worley, Raper, and Hohl 1979).

\section{Sorodiplophrys (Stramenopiles)}

Little has been published about Sorodiplophrys stercorea (Dykstra and Olive 1975; Tice et al. 2016). Similar to other sorocarpic amoebae, however, single-celled amoebae aggregate 
and form a fruiting body. The final fruiting body structure consists of a small, thick stalk composed of secreted material and dead cells that supports a spherical, golden, mucoid sorus (Fig. 1E). The presence of dead stalk cells indicates that this organism shows reproductive division of labor as well.

Finally, while many examples of aggregative multicellular fruiting body formation involve sorocarpic amoebae, it is worth noting that there are additional non-amoeboid taxa that undergo cooperative fruiting.

Myxobacteria are a clade of prokaryotic organisms that undergo aggregative fruiting in response to starvation. Their fruiting bodies can vary dramatically among different species. For example, some species form clear stalks that lift up the spores, whereas in others, the stalk is reduced or absent (Velicer and Vos 2009). The best-known species is Myxococcus xanthus, which has been used as a model system for cooperation and conflict (see chapter by [Velicer and colleagues]). The life cycle of $M$. xanthus is similar to that of social amoebae: the soil-dwelling bacteria prey upon other microbes, sometimes cooperatively as a swarm (Mauriello et al. 2010). Upon starvation, the bacteria aggregate into mounds and form fruiting bodies, where only a fraction of the cells differentiates into spores, and others either remain as rod-shaped cells or undergo autolysis (Fig. 1F) (Nariya and Inouye 2008; Varon, Cohen, and Rosenberg 1984). The percentage of cells that become viable spores is much lower than in some of the other eukaryotic species discussed so far, with a non-spore percentage of up to $90 \%$ in M. xanthus, at least under laboratory conditions (Velicer and Vos 2009). The reason for the variation in fruiting body morphology among species, including in the formation of a stalk, is not well understood (Velicer and Vos 2009).

Sorogena stoianovitchae (eukaryotic supergroup Alveolata) is unique among ciliates in undergoing aggregative fruiting (L. S. Olive and Blanton 1980; Sugimoto and Endoh 2006). In its unicellular stage, it feeds on other ciliate species. Upon food shortage, however, it 
aggregates beneath the water surface and forms an aerial fruiting body. The stalk is produced via collective secretion of a mucous material by the entire population. The stalk lifts the population out of the water, after which each of the cells become encysted and together form a sorus (Sugimoto and Endoh 2006).

\section{Fruiting Body Formation in the Amoebozoa}

Within the Amoebozoa, the group historically known as Eumycetozoa (true slime molds) consisted of three major classes of organisms: Protostelids, Myxogastrids, and Dictyostelids. The latter two groups are monophyletic, whereas molecular analyses indicate that protostelids are not. For this reason, they are now sometimes referred to as "protostelioid amoebae" rather than "protostelids", to emphasise common elements of their morphology in lieu of a phylogenetic classification (Shadwick et al. 2009). Protostelioid amoebae undergo sporocarpic development (Spiegel et al. 2017). The amoeba secretes an extracellular matrix, which forms a stalk that lifts the amoeba up. The amoeba then differentiates into a spore, sometimes following cell division (Furtado and Olive 1971; Spiegel et al. 2017; Lahr et al. 2011). Thus, sporocarpy in protostelids results in the production of microscopic fruiting bodies that contain only one or a few cells.

The plasmodial slime molds belong to the monophyletic group Mxyogastrea, another taxon of sporocarpic amoeba. Plasmodial slime molds are named for the slimy structure they produce—called a plasmodium—which forms when amoeboid cells undergo repeated rounds of mitosis without cytokinesis. This process results in a single, massive, multinucleated cell with a continuous cytoplasm, which can reach many meters in size. When conditions turn bad, the plasmodium produces masses of stalked fruiting bodies, a process that occurs not through growth, but through rearrangement of the existing biomass (Stephenson and Schnittler 2017). Thus, they form fruiting bodies, albeit not achieved through aggregation. Spores are mostly wind-dispersed from the fruiting bodies and 
germinate to form the plasmodium again. Plasmodial slime molds were the likely inspiration for the 1950's horror film The Blob. Like protostelids, the stalks produced by plasmodial slime molds are acellular (i.e., secreted). Unlike the protostelids, however, their fruiting bodies are macroscopic.

Finally, the dictyostelids consists of more than 160 species (Romeralo et al. 2011). Their phylogenetic tree contains many long, unbroken branches, which suggests that they have been undersampled and that the true diversity of the group is even greater (Romeralo et al. 2011). Although the phylogeny has been revised over the years, recent phylogenies based on SSU rRNA and alpha-tubulin sequences group dictyostelids into two major clades, the Dictyosteliales and the Acytosteliales, each of which is composed of two groups-resulting in groups 1-4, referred to below. These groups are then further subdivided (e.g., into groups 2A and 2B) (Romeralo et al. 2011). The model organism D. discoideum, discussed in detail below, belongs to group 4 .

Comparative analyses indicate that formation of a stalked fruiting body is conserved within dictyostelids (Schaap et al. 2006; Sucgang et al. 2011; Heidel et al. 2011; Romeralo et al. 2011). Romeralo et al. (2013) combined genetic data from 99 species with the phenotypic data of 24 traits in each of these species. This work suggests that their last common ancestor ( 0.6-1.0 billion years ago) formed fruiting body structures that lift spores up in the air. However, as we emphasise below, the species show substantial variation in the formation and appearance of their fruiting bodies (Fig. 1). The evolutionary drivers of such diversity in fruiting structures are still being investigated. Nevertheless, this diversity makes this group suitable to study the function of a stalk, its associated costs and benefits, and the possible functional constraints on this structure. 


\section{Morphological Variation Among Dictyostelids, with Emphasis on Stalk Formation}

In this section, we discuss fruiting body formation and function from the perspective of the model organism $D$. discoideum. We start by describing the life cycle and stalk formation in $D$. discoideum. We then describe some of the morphological variation within dictyostelids, particularly in when and how they form their stalked fruiting bodies.

Dictyostelium discoideum is a soil-dwelling amoeba frequently isolated from the upper layer of the soil of mostly deciduous forests located in the temperate zone (Swanson, Vadell, and Cavender 1999; Landolt, Stephenson, and Cavender 2006). Its life cycle is broadly similar to many of the sorocarpic amoebae described in previous sections. However, its developmental cycle is more complex, as it involves division of labor, extensive cell-cell communication and coordinated cell death. $D$. discoideum initiates aggregation in response to deteriorating environmental conditions. Starving cells cease phagocytosis, secrete extracellular cyclic adenosine monophosphate (cAMP), and respond chemotactically to sources of cAMP, which results in cell streaming and aggregation to form a mound. In the mound, cells initially differentiate into either pre-stalk or pre-spore cells, indicative of their eventual cell fates in the later fruiting body (see Figure 2 of Schaap chapter). The pre-spore cells move to the top of the mound, which forms a tip that elongates into a finger-like structure that falls to the surface. The resulting worm-like structure, called a slug, migrates away from the site of aggregation. Following migration, and upon detecting cues such as overhead light, the slug transforms into a multicellular fruiting body. Cells at the anterior of the slug undergo apoptosis, having vacuolised and hardened to form a rigid, dead cellular stalk. The remaining cells move to the top of the stalk and differentiate into viable spores, which disperse and germinate to release single-celled amoebae (Fig. 1G). In addition to this multicellular stage, which results in asexually produced spores, $D$. discoideum has a sexual stage. The sexual stage also involves aggregation and cell sacrifice (through cannibalism). It results in the formation of a durable structure, called a macrocyst, that is not stalked 
(Bloomfield 2010; Bloomfield 2011; see also Schaap chapter). Meiosis takes place during the formation of the macrocyst, and the amoebae that later emerge are recombinants.

D. discoideum is a model system for cell biology, developmental biology, chemotaxis, and host-pathogen interactions (reviewed in Bozzaro 2019; Williams 2010). It is genetically tractable, has a precise 24-hour development cycle, and terminal differentiation results in a small number of distinct cell types. $D$. discoideum is also notable for its relatively stable celltype proportions: approximately $80 \%$ of cells in the posterior of the slug will form the sporecontaining sorus, whereas $\sim 20 \%$ of cells in the anterior die to form the stalk. These cell-type proportions can partially re-establish following perturbations, for example, by ablation of either the anterior (prestalk) or posterior (prespore) sections of the slug (Ràfols et al. 2001; Raper 1940). The robustness of its spore-stalk cell proportions is of interest to developmental biologists interested in how multicellular organisms achieve and maintain specific cell-type proportions, as well as evolutionary biologists interested in whether and how an altruistic stalk can be maintained.

While $D$. discoideum is by far the best studied of the social amoebae species, it exhibits a variety of traits that are somewhat uncommon among dictyostelids. Below, we focus on three morphological traits, namely stalk composition, stalked migration and clustering and branching patterns, comparing $D$. discoideum to other species. Although it is difficult to ascertain the adaptive significance of this variation in morphology, we discuss functional implications of the different structures and some of their potential costs and benefits.

\section{Cellular vs Acellular Stalks}

Aggregative multicellularity—by virtue of allowing unrelated cells to collaborate to form a multicellular individual—presents opportunities for conflict, especially if there are different fitness costs and benefits associated with adoption of different cell fates. This problem is particularly severe in social amoebae, where cells that form the stalk will die and the 
remainder will live, providing a large fitness advantage to strains that can avoid the stalk fate. Conflict is thought to emerge over which cells will adopt the dead-cell fate and which will survive into the next generation. The opportunity for different genotypes to co-aggregate means that selection has the opportunity to favour genotypes that behave selfishly (Ostrowski 2019).

Stalk formation does not necessarily require self-sacrifice. The acytostelids (Group 2A), for example, form acellular stalks, consisting of a hollow tube that is made from secreted cellulose, with all cells subsequently forming viable spores atop the stalk (Mohri et al. 2013). In contrast, cellular stalks are made from an inner layer of hardened vacuolized stalk cells and an outer layer of cellulose (Gezelius 1959). Why some species evolved to use cellular stalks, whose formation depends on the death of a fraction of the population, while others form stalked fruiting bodies without such a sacrifice, is not known.

At present, we can only speculate about why these differences might have evolved. One possibility is that cellular stalks might provide stronger support, allowing larger aggregate sizes and taller stalks that can support more spores. For example, acytostelids have a smaller aggregate size and form smaller structures compared to species with cellular stalk formation (170-1200 mm versus $1200-8200 \mathrm{~mm}$ ), which might be consistent with a weaker stalk in the former (Raper 1956b; Schaap et al. 2006). Additionally, Kaushik and Nanjundiah (2003) point out that production of an acellular stalk might be energetically costly to the cells and therefore detract from their ability to survive for long periods. The division of labor achieved through formation of a cellular stalk might entail benefits for the survival of the spores.

One possible benefit of acellular stalk formation is the ability to produce a fruiting body with a smaller population. This potential benefit is apparent in Dictyostelium lacteum, the only species known to be capable of producing both cellular and acellular stalks. When food 
availability is low, the species forms a small, acellular stalk, similar to that produced by acytostelids; only at higher cell numbers is a larger, cellular stalk formed (Bonner and Dodd 1962; Bonner 2006). While D. lacteum is the only species known to be plastic for cellular stalk formation, it is possible that other dictyostelids possess similar plasticity but remain to be discovered, or that their plasticity has simply not been noticed. Finally, although some have speculated that acytostelids could represent an intermediate stage in the evolutionary transition from simple (e.g., single cell type, no division of labor) to complex (multiple cell types with division of labor) multicellularity (Olive 1975; Bonner 2003), a molecular study by Romeralo (2013) concluded that the most recent common ancestor of the dictyostelids likely already displayed cellular stalk formation, suggesting that acellular stalk formation is a derived trait.

\section{Stalked migration}

In some species, aggregation of cells is followed by the formation of a slug that migrates away from the point of aggregation (Fig. 2). For example, in D. discoideum, the slug forms approximately $12 \mathrm{hr}$ after the onset of starvation, and it can travel long distances from the site of aggregation, resulting in movement of up to $6 \mathrm{~cm}$ in a week (Jack et al. 2015; Jack et al. 2011). In D. discoideum, the slugs are strongly phototactic, moving towards a directional light source. Migration ceases once light is overhead, which triggers culmination to form a fruiting body. The combination of attractants (light and heat) and repellents (high ammonia levels) is thought to direct slugs upwards through the soil, into an open area suitable for fruiting body formation (Raper 1984; Bonner and Lamont 2005).

Slug migration is thought to have evolved several times in the major groups of the dictyostelids (Romeralo et al. 2013). In D. discoideum, the stalk is not formed until after slug migration, during the final stages of development. However, in the majority of dictyostelid species, stalk is continuously produced from the rear of the slug during its migration (Fig. 2B). Ancestral trait reconstruction suggests that the last common ancestor of the 
dictyostelids was likely a stalked migrator, with only a few species $(D$. discoideum, $D$. polycephalum, $D$. citrinium, $D$. intermedium and $D$. dimigraformum) having evolved stalkless migration (Schaap et al. 2006; Schaap 2007; Romeralo et al. 2013).

Several studies have addressed the potential costs and benefits of stalked versus stalkless migration (Bonner 1982; Gadagkar and Bonner 1994; Jack et al. 2011). Jack et al. (2011) quantified the costs of slug migration in $D$. purpureum (a stalked migrating species) and $D$. discoideum (which does not undergo stalked migration). Both species showed a tradeoff between migration and sporulation, as they show a similar decrease in sporulation after controlling for differences in migration distance. The authors suggest that the two species could have adapted their behavior to different stages of the life cycle. As $D$. purpureum produces much taller fruiting bodies than $D$. discoideum (>7 mm versus 3-7 $\mathrm{mm}$ ), the authors suggest that $D$. purpureum invests less in migration (active dispersal) and more in fruiting body size (passive dispersal). In contrast, $D$. discoideum invests relatively more in active dispersal so it can reach suitable fruiting locations further away, though it produces a shorter fruiting body structure.

That the stalk might serve a different or additional purpose in different dictyostelids was proposed by Bonner (1982) and later tested by Gilbert et al. (2012). They showed that the stalked migrator $D$. giganteum could use its stalk as a bridge to traverse small gaps in the substratum. In contrast, slugs of stalkless migrator $D$. discoideum were not able to traverse these same gaps, suggesting one potential advantage of stalked migration. Regardless of some of the potential benefits of stalked migration, it is surprising that cells might coaggregate to form a slug with no guarantee as to how large of a stalk-and thus, how big of a cell sacrifice-will be made.

Because the costs of stalk formation are likely to be greater for stalked migrators like $D$. purpureum, one might expect that higher levels of relatedness within cooperative fruiting 
bodies would likewise need to be greater. Indeed, while both $D$. discoideum and $D$. purpureum possess mechanisms of kin discrimination, and thus imperfectly separate out during chimeric development (Mehdiabadi et al. 2006; Ostrowski et al. 2008), D. purpureum seems to segregate more completely than $D$. discoideum. It would be interesting to know whether $D$. purpureum has a stronger history of selection on the genes that underpin its kin discrimination. These genes have been identified in D. discoideum (Hirose et al. 2011), but are not yet known in $D$. purpureum.

\section{Clustering and branching patterns}

In $D$. discoideum, each aggregate gives rise to a fruiting body, which consists of a relatively thick, non-branched stalk that holds aloft a single sorus. This morphology is common among species in Group 4, but outside of this group there is a large variety of structures that differ in their degree of clustering and branching (Fig. 3). In some species, secondary tips form after aggregation, giving rise to multiple, closely spaced fruiting bodies (referred to as "gregarious" development) or fruiting body structures with multiple sori emanating from one stalk ("like flowers in a vase”, Raper 1956). Fruiting bodies can also be branched and/or consist of whorls (Schaap et al. 2006; Baldauf and Strassmann 2017). The extent of the branching and clustering is also plastic, as it can depend on cell density (Bonner and Dodd 1962; Romeralo et al. 2013). The general pattern was that larger structures-i.e., branched and clusteredtend to form in response to high cell density, whereas unbranched and solitary structures would emerge at low cell density.

\section{Altruism, Stalk Formation, and the Maintenance of Multicellularity in D. discoideum} In previous sections, we discussed numerous examples of aggregative multicellularity that result in the formation of a stalked fruiting body. We emphasized that, in many cases, many or all of the cells in the fruiting body remain viable, although there could be fitness costs associated with exactly which role is adopted by a given cell. We also emphasised that some species form fruiting bodies with secreted, non-cellular stalks, and others form extensive 
cellular stalks throughout migration, resulting in a potentially large and unpredictable cell sacrifice.

The formation of cellular stalks by dictyostelids is of special interest to evolutionary biologists. Stalk formation is likely altruistic, in that some give up opportunities for direct fitness in order to form a structure that appears to benefit the rest. This differentiation into spore and stalk cells is analogous to the differentiation into soma and germline that is seen in complex multicellularity. Stalk formation is a clear example of reproductive division of labor, where some cells specialise in reproduction, and others specialise in non-reproductive functions.

Certain features of the Dictyostelium life cycle mean that its reproductive division of labor might be evolutionarily fragile. Aggregative multicellularity potentially permits multiple different genotypes to co-aggregate and form chimeric multicellular structures. This genetic diversity in combination with strong fitness consequences for becoming stalk vs spore means that natural selection can operate during this stage of the life cycle. Thus, all else being equal (and it may not be), natural selection should favor genetic variants that can avoid the costly role of the stalk and disproportionately adopt the high-fitness spore fate. The problems posed by chimerism in Dictyostelium and its potential consequences for the evolution of multicellularity are discussed more fully in the chapter by Jahan et al. Given these opportunities for selection to favor stalk-avoiders, one long-standing question is the extent to which opportunities for conflict may have influenced how multicellularity evolves (e.g., whether aggregative multicellularity is successful) and whether it is evolutionarily stable. In Dictyostelium, this possibility has led to interest in whether stalkless forms might evolve in nature. 


\section{Hunting for Stalkless Strains in Nature}

There have been limited attempts to find stalkess strains in nature. Buss (1982) reported the existence of a stalkless Dictyostelium strain. While growing and isolating Dictyostelium fruiting bodies from soil samples, he observed two distinct fruiting body morphologies. One strain showed a standard stalked morphology, whereas the other produced a ball of spores directly on the substratum, without a stalk. Buss described the latter strain as a "somatic cell parasite", because it was capable of "reproducing itself and spreading infectiously" at the costs of the rest of the population (Buss 1982). To study whether such a stalkless strain could be maintained in the population over multiple generations, he co-cultured the stalked and stalkless strain over a number of generations. The stalkless strain increased in frequency from rarity and, at a sufficiently high starting ratio, it could become fixed. However, when the stalkless strain was mixed with a different set of strains isolated from the same soil sample, no chimeric fruiting bodies were observed, indicative of some sort of recognition mechanism present in the other strains able to distinguish self from non-self. Unfortunately, the stalkless strain was subsequently lost (Buss, pers. communication), making it impossible to study it further. This means that it is not possible to carry out molecular analyses to establish which dictyostelid species it was or to identify the genetic changes responsible for its unusual morphology.

More recently, Gilbert et al. (2007) attempted to isolate stalkless strains from natural populations. Unfortunately, dictyostelid fruiting bodies are too small and infrequent to find simply by directly examining soil samples. Moreover, finding stalkless strains—given that they do not form the recognizable macroscopic fruiting body-is even more challenging. However, previous work showed that fruiting bodies can sometimes be observed on animal dung pellets (Stephenson and Landolt 1992; Raper 1984). Gilbert and colleagues collected dung pellets, brought them back to the lab, and incubated them with or without additional bacteria for food until fruiting bodies formed (Gilbert et al. 2007). They then collected the spores from 95 of these fruiting bodies and plated them at low density on a lawn of bacteria. 
Under these conditions, well-spaced spores germinate and divide to produce circular plaques-clearings in the bacterial lawn where the amoebae have devoured the prey. At the center of each plaque, where the amoeba cell density is high and food has been depleted, fruiting bodies will form if the strain is capable of multicellularity. Thus, dilution plating of spores to see whether they give rise to fruiting bodies is a way to screen for the presence of strains that have lost multicellularity, yet previously managed to join and form a stalked fruiting body with others, or that exhibit other morphological alterations. However, despite screening $>3,300$ plaques, Gilbert and colleagues observed no stalkless morphologies.

To our knowledge, the works by Buss (1982) and Gilbert et al. (2007) are the only studies that have attempted to identify and/or quantify the frequency of stalkless strains in nature. In the future, as new methods for single-cell genomics improve, it might be possible to use culture-independent methods to isolate, sequence, and identify each amoeba cell in a soil sample, enabling identification of natural isolates that have lost multicellular development or evolved novel morphologies not currently recognized. For now, however, whether $D$. discoideum's aggregative fruiting can lead to selfishness that threatens the maintenance of the stalk in nature remains unknown.

\section{Laboratory and Theoretical Studies of Stalklessness}

The observation by Buss (1982) of a stalkless morphology motivated laboratory studies of stalk-avoiding mutants as well as theory to address the circumstances under which stalklessness might evolve and persist. For example, two studies examined an insertion mutant (fbxA-mutant) that contributes less to the stalk in chimerae with the wild-type strain (Nelson et al. 2000; Ennis et al. 2000). When developed clonally, the mutant forms aberrant fruiting bodies that contain few to no spores or fail to initiate stalk production altogether (Ennis et al. 2000; Gilbert et al. 2007). However, when co-developed with a wild-type strain, it produces a disproportionate fraction of the spores. Ennis and colleagues (2000) speculated that the $f b x A$ gene takes part in the regulation of a complex involved in the 
differentiation in spore and stalk cells, where deletion of the gene causes the stalk cell differentiation pathway to be halted.

Gilbert and colleagues (2007) subsequently used the $f b x A$ - mutant to examine the extent to which such a strain that does not contribute fairly to the stalk might increase in frequency in a population owing to its advantage in spore production in chimerae. However, such a strain may face a disadvantage at high frequency, if it has displaced the very strain it relies on to sporulate. The impacts of these different frequencies can be quantified as relatedness, which encompasses the degree to which the mutant interacts with self $(r=1)$ or with the stalk-proficient wild-type $(r=0)$ to build a fruiting body. The authors found that when $r>0.75$, the $f b x A$ - mutant decreased in frequency, indicating net selection against the mutant. This work demonstrated that sufficiently high relatedness could be essential for preventing the invasion and takeover of populations by non-stalk-forming strains.

The above empirical examples suggest that stalk-avoiding strains, provided that the behavior is costly in the absence of a cooperating strain, could be selected against when relatedness is high—but is relatedness in natural populations sufficiently high to accomplish this? Relatively little is known about relatedness in nature, especially over the small spatial scales in soil where different strains might encounter one another and co-develop to form chimeric fruiting bodies. Fortunato and colleagues genotyped natural isolates from minute soil samples collected using a plastic straw with a diameter of $6 \mathrm{~mm}$ (Fortunato et al. 2003). Of 26 soil samples that contained Dictyostelium, $63 \%$ yielded more than one genotype, with as many as 9 distinct genotypes from a single soil sample. These results yielded an estimate of average genetic relatedness of 0.52 . In addition, Gilbert collected and genotyped 88 individual fruiting bodies that emerged from 25 dung piles incubated in the lab (Gilbert et al. 2007). Seventy-seven percent of the fruiting bodies contained only a single genotype, which yielded a minimum relatedness of $r=0.86$-high enough to support their hypothesis that 
relatedness would be high enough to select against the $f b x A$ - mutation in nature (i.e., $0.86>0.75)$.

The hypothesis that high genetic relatedness would be sufficient to stop the spread of nonstalked mutants was further supported by Kuzdzal-Fick et al. (2011). They carried out laboratory evolution experiments that involved multicellular development under either high or low relatedness conditions (i.e., in genetically diverse or clonal groups, respectively). Approximately one-third of populations evolved at low relatedness harbored strains that could not form a stalked fruiting body when developed clonally but were disproportionately represented among the spores when co-developed with their ancestor. Conversely, no losses of multicellularity occurred in the high relatedness experiment. Taken together, these experiments collectively support that high relatedness is an important condition for the maintenance of the stalked fruiting body in this organism.

Hudson et al. (2002) developed a mathematical model that sought to address the evolutionary stability of stalk formation. This model made two assumptions about how stalks influence fitness: (1) that the fitness of a stalkless strain would be low and (2) that dispersal success increases with stalk size. They showed that stalk formation could still be maintained in populations founded by genetically unrelated individuals. However, they also showed that the presence of selfish genotypes, which contribute less to the stalk, could drive the evolution of suboptimal stalk sizes-that is, reductions in the allocation to the stalk relative to what would be optimal, assuming that dispersal is an important fitness component. Similarly, a model by Brännström and Dieckmann (2005) supported the potential for coexistence of multiple genotypes (e.g., stalked and stalkless) within a single population. Taken together, these experiments suggest that one way to identify stalkless strains in nature might be to look for them under natural conditions where relatedness is low (i.e., genetic diversity is high) or the importance of dispersal is low. Moreover, while it remains unknown how essential stalk formation is for dispersal per se (as opposed to simply protecting the spores 
by lifting them in the air), one study did show that spores from an intact, stalked fruiting body were more likely to be acquired by an insect vector than those that were placed directly on the substrate; the spores also survived passage in the insect gut when ingested (smith, Queller, and Strassmann 2014). This finding thus provides some support for the role of the stalk in facilitating spore dispersal.

\section{Naturally Occurring Variation in Stalk Size}

The model by Hudson et al. (2002) suggested that the size of the stalk in $D$. discoideum could reflect a tradeoff between its cost to the cells that die and its benefit to the surviving spores, and thus that its size may be sub-optimal, at least for dispersal. Similarly, the study by Brännström and Dieckmann (2005) supported the idea that within-population polymorphisms could arise, such that we might expect to observe natural variation in the degree of stalk investment. To what extent is there evidence that this variation exists?

Within $D$. discoideum, only a few studies have looked at natural variation in overall stalk or fruiting body size, as well as equity of spore-stalk allocation between strains (Buttery et al. 2009; Votaw and Ostrowski 2017). Buttery et al. (2009) estimated spore-stalk allocation alone and when strains were co-developed in pairwise combinations for six strains from a site in North Carolina. Spore allocation was estimated in two ways: from images of individual fruiting bodies, followed by estimation of relative sorus and stalk volume, and by comparing the spore production of strains clonally and following development in pairwise chimerae, under the assumption that more spores indicates less allocation to stalk. Using the latter approach, they observed variation in spore allocation (up to 2.8-fold difference) among strains (Buttery et al. 2009).

Ostrowski and Votaw (2017) looked at variation among strains, among sites, and in clonal versus pairwise chimerae using strains from two geographically distant sites, one in North Carolina and one in Texas. In addition to imaging and measuring stalk height and spore 
number, GFP reporter strains were used to estimate spore-stalk allocation. They observed variation among strains within both populations in stalk allocation, but also larger size overall for Texas strains compared to those from North Carolina. These results underscore that increases in stalk size can be accomplished through two routes: either by allocating a higher fraction of cells to the stalk or by forming larger fruiting bodies altogether, presumably through production of fewer, larger aggregates from the same starting cell number. In addition, after controlling for differences in overall fruiting body size, within-population variation in relative stalk size was observed in both populations, similar to the findings by Buttery et al. (2009). These studies together indicate that some polymorphism in clonal and chimeric spore-stalk allocation occurs among strains within a given site, but also that the morphology of the fruiting bodies can evolve divergently among locations.

Overall, the studies of altruistic stalk formation by $D$. discoideum, clonally and in chimerae, illustrate the potential for conflict in organisms that undergo aggregative multicellularity with division of labor. Aggregative multicellularity can lead to genetic diversity within the multicellular organism, providing the fuel for natural selection, whereas division of labor generates the strong competitive advantages to cells that avoid the altruistic fate. Studies in this organism thus help to validate the predictions of evolutionary theory about the problems of aggregative multicellularity.

\section{Conclusions}

Aggregative fruiting has independently evolved in five of the six supergroups of the Eukarya, suggesting that the formation of a stalk is a morphological adaptation. Nevertheless, there is a substantial diversity in how these structures are formed-whereas some stalks are composed of dead cells (and thus involve cell sacrifice), others form by secretion, such that all cells potentially survive as spores. Differences in how stalks are formed in organisms that undergo aggregative multicellularity has important implications for the evolutionary maintenance of this trait. 
Substantial variation in how stalked fruiting bodies form exists within dictyostelids alone, a large clade consisting of more than 150 species within the Amoebozoa. Here we described variation among species in the composition of the stalk (acellular versus cellular), the timing of its production (during or after migration), and its branching morphology (branched or unbranched, whorled or not). Unfortunately, the explanations for the variation in these features are not known, although several studies provide information about some of the potential functions of the stalk. For example, spores that sat atop stalks were more likely to be picked up by an insect vector (smith, Queller, and Strassman 2014), and stalks emanating from the rear of the slug can help in traversing gaps in the soil (Gilbert et al. 2012) - yet, whether and how these features are used in nature remains to be seen. In addition, while some stalk features may provide a fitness advantage, there may also be functional constraints imposed by development or physics. For example, acellular stalks have the benefit of not necessitating cell sacrifice, but these structures may not support as many spores, or the spores may be of lower quality (Kaushik and Nanjundiah 2003).

In those species that form cellular stalks, death of a fraction of the cells presents opportunities for conflict, as strains that avoid forming the stalk and disproportionately form spores should have a fitness advantage. In the model organism $D$. discoideum, studies of stalk-avoiding mutants and the behaviors of natural isolates, as well as mathematical models have all contributed to our understanding of the evolutionary maintenance of altruistic stalk formation. These studies confirm an essential role for relatedness, but whether relatedness is high enough in nature to prevent takeover by stalk-avoiding strains remains uncertain. Future studies would benefit from consideration of how the evolution of morphology has been impacted by variation in relatedness across populations, as well as of other factors that might also promote the evolutionary maintenance of cooperative multicellularity. 
Figure Legends

Figure 1. Fruiting body morphologies throughout the eukaryotic tree of life. (A)

Guttulinopis - Rhizaria, (B) Acrasis rosea - Excavata, (C) Copromyxa protea - Amoebozoa,

(D) Fonticula alba - Opisthokonta, (E) Sorodiplophrys stercorea - Stramenopiles, (F)

Sorogena stoianovitchae - Alveolata, (G) Dictyostelium discoideum - Amoebozoa. Fruiting bodies are not drawn to scale.

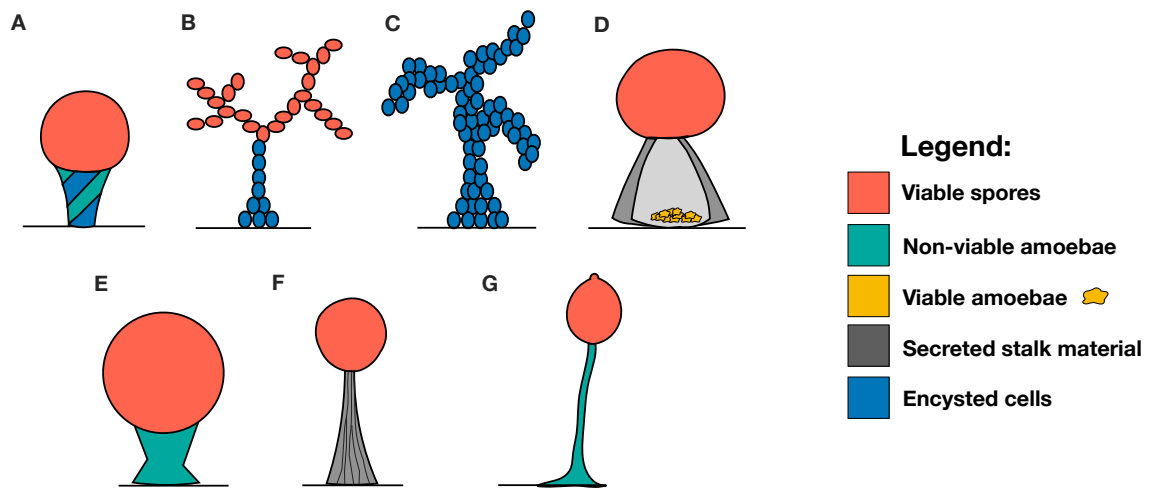


Figure 2. Stalked versus stalkless migration among dictyostelid species. (A) D. discoideum and (B) D. purpureum, both Group 4 species. D. discoideum has stalkless migration and an upright, sturdy stalk with a flattened disk of cells at its base. Its slugs lie on the substrate, with the tip (pre-stalk zone) in the air. It produces yellow sori. D. purpureum has stalked migration, produces a slender stalk that lacks a basal disc, and its sori are purple. (C) D. discoideum and D. purpureum co-occurring on the same soil isolation plate. The pale yellow fruiting bodies are $D$. discoideum, whereas the dark fruiting bodies are $D$. purpureum. (D) A different soil isolation plate, showing an unidentified dictyostelid species with stalked migration.
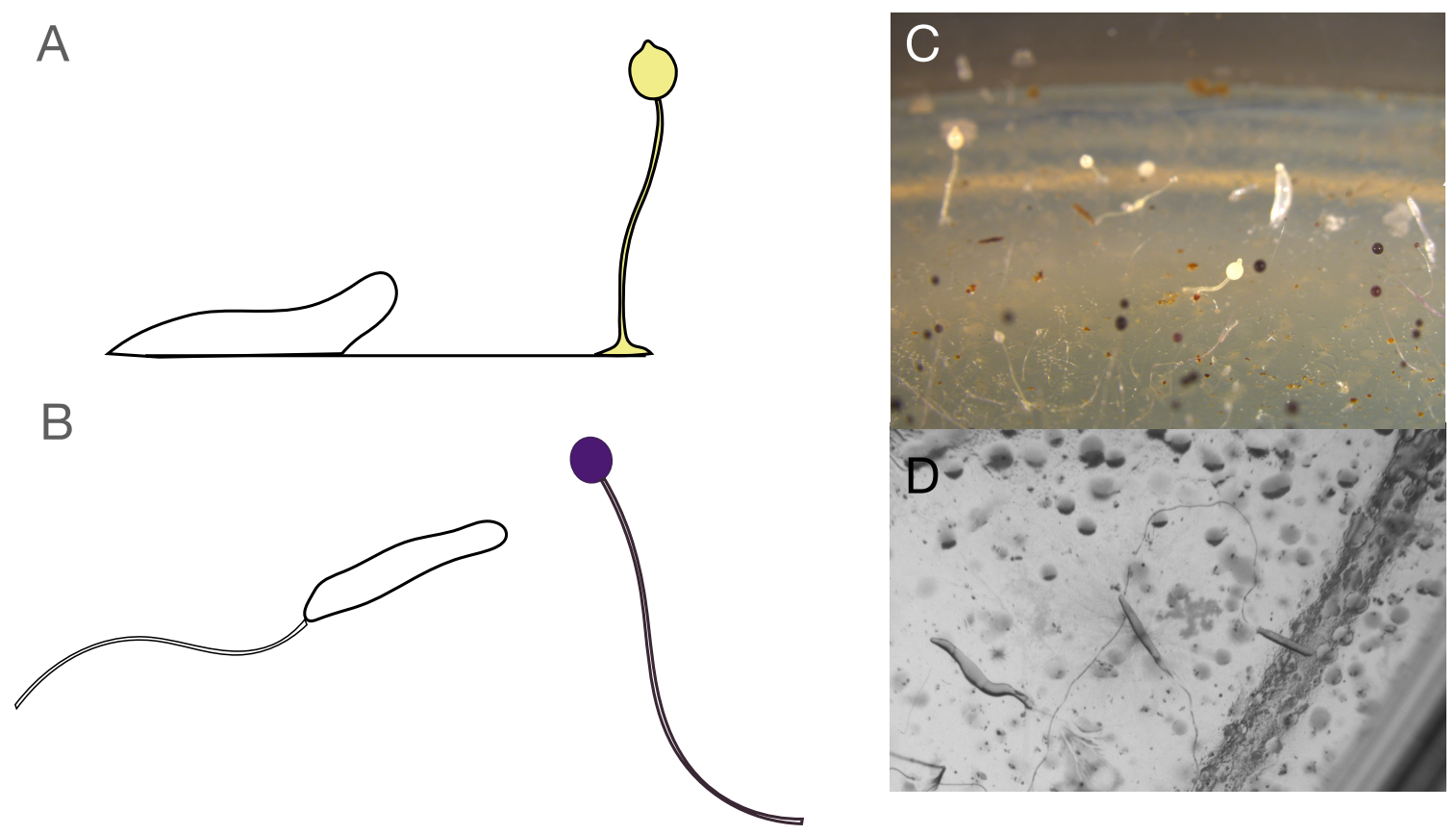
Figure 3. Examples of clustering and branching patterns in the fruiting bodies of protostelids and dictyostelids. Approximate fruiting body height is indicated below each species, based on descriptions in Raper (1984), except for $P$. violaceum, where the value listed is the mean stalk length from Romeralo et al. (2013). Note that fruiting body size can vary substantially depending on plating conditions, so only an approximate range is provided.

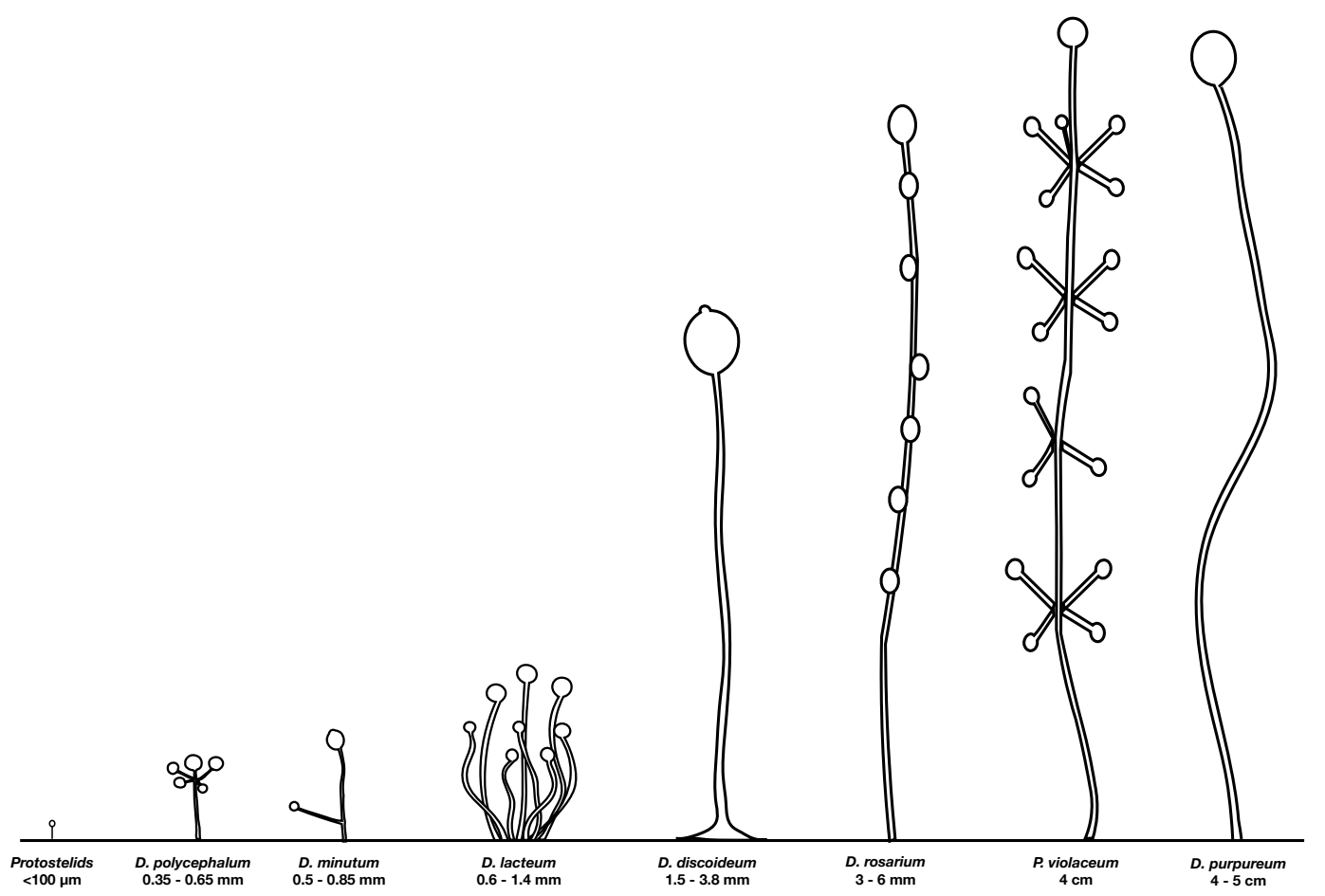




\section{References}

Bloomfield, Gareth. 2011. "Genetics of Sex Determination in the Social Amoebae." Development, Growth \& Differentiation 53 (4): 608-16.

Bloomfield, Gareth, Jason Skelton, Alasdair Ivens, Yoshimasa Tanaka, and Robert R. Kay. 2010.

"Sex Determination in the Social Amoeba Dictyostelium Discoideum." Science 330 (6010): 153336.

Bonner, John Tyler, and Marya R. Dodd. 1962. "Aggregation Territories in the Cellular Slime Molds."

The Biological Bulletin 122 (1). https://doi.org/10.2307/1539317.

Bonner, J. T. 1982. "Evolutionary Strategies and Developmental Constraints in the Cellular Slime Molds." The American Naturalist 119 (4): 530-52.

Bowler, Diana E., and Tim G. Benton. 2005. "Causes and Consequences of Animal Dispersal Strategies: Relating Individual Behaviour to Spatial Dynamics." Biological Reviews of the Cambridge Philosophical Society 80 (2): 205-25.

Bozzaro, Salvatore. 2019. "The Past, Present and Future of Dictyostelium as a Model System." The International Journal of Developmental Biology 63 (8-9-10): 321-31.

Brännström, A., and U. Dieckmann. 2005. "Evolutionary Dynamics of Altruism and Cheating among Social Amoebas." Proceedings. Biological Sciences / The Royal Society 272 (1572): 1609-16.

Brefeld, O. 1869. "Ein Neuer Organismus Aus Der Verwandtschaft Der Myxomyceten." Abhandlungen Der Senckenbergischen Naturforschenden Gesellschaft Frankfurt 7: 85-107.

Brown, Matthew W., Martin Kolisko, Jeffrey D. Silberman, and Andrew J. Roger. 2012. "Aggregative Multicellularity Evolved Independently in the Eukaryotic Supergroup Rhizaria." Current Biology: CB 22 (12): 1123-27.

Brown, Matthew W., and Jeffrey D. Silberman. 2013. "The Non-Dictyostelid Sorocarpic Amoebae." In Dictyostelids: Evolution, Genomics and Cell Biology, edited by Maria Romeralo, Sandra Baldauf, and Ricardo Escalante, 219-42. Berlin, Heidelberg: Springer Berlin Heidelberg.

Brown, Matthew W., Jeffrey D. Silberman, and Frederick W. Spiegel. 2011. "'Slime Molds' among the Tubulinea (Amoebozoa): Molecular Systematics and Taxonomy of Copromyxa." Protist 162 (2): $277-87$.

Brown, Matthew W., Frederick W. Spiegel, and Jeffrey D. Silberman. 2009. "Phylogeny of the 'Forgotten' Cellular Slime Mold, Fonticula Alba, Reveals a Key Evolutionary Branch within 
Opisthokonta." Molecular Biology and Evolution 26 (12): 2699-2709.

Buss, L. W. 1982. "Somatic Cell Parasitism and the Evolution of Somatic Tissue Compatibility."

Proceedings of the National Academy of Sciences of the United States of America 79 (17): $5337-$ 41.

Buttery, Neil J., Daniel E. Rozen, Jason B. Wolf, and Christopher R. L. Thompson. 2009.

"Quantification of Social Behavior in D. Discoideum Reveals Complex Fixed and Facultative Strategies." Current Biology: CB 19 (16): 1373-77.

Dykstra, Michael J., and Lindsay S. Olive. 1975. "Sorodiplophrys: An Unusual Sorocarp-Producing Protist." Mycologia 67 (4): 873-79.

Ennis, H. L., D. N. Dao, S. U. Pukatzki, and R. H. Kessin. 2000. "Dictyostelium Amoebae Lacking an F-Box Protein Form Spores rather than Stalk in Chimeras with Wild Type." Proceedings of the National Academy of Sciences of the United States of America 97 (7): 3292-97.

Fisher, R. M., J. Z. Shik, and J. J. Boomsma. 2020. "The Evolution of Multicellular Complexity: The Role of Relatedness and Environmental Constraints." Proceedings. Biological Sciences / The Royal Society 287 (1931): 20192963.

Fortunato, A., J. E. Strassmann, L. Santorelli, and D. C. Queller. 2003. "Co-Occurrence in Nature of Different Clones of the Social Amoeba, Dictyostelium Discoideum." Molecular Ecology 12 (4): 1031-38.

Furtado, J. S., and L. S. Olive. 1971. "Ultrastructural Evidence of Meiosis in Ceratiomyxa Fruticulosa." Mycologia 63 (2): 413-16.

Gadagkar, Raghavendra, and J. Bonner. 1994. "Social Insects and Social Amoebae." Journal of Biosciences. https://doi.org/10.1007/bf02703057.

Gezelius, K. 1959. "The Ultrastructure of Cells and Cellulose Membranes in Acrasiae." Experimental Cell Research 18 (November): 425-53.

Gilbert, Owen M., Kevin R. Foster, Natasha J. Mehdiabadi, Joan E. Strassmann, and David C.

Queller. 2007. "High Relatedness Maintains Multicellular Cooperation in a Social Amoeba by Controlling Cheater Mutants." Proceedings of the National Academy of Sciences of the United States of America 104 (21): 8913-17.

Gilbert, Owen M., Jennie J. Kuzdzal-Fick, David C. Queller, and Joan E. Strassmann. 2012. "Mind the Gap: A Comparative Study of Migratory Behavior in Social Amoebae." Behavioral Ecology and 
Sociobiology 66 (9): 1291-96.

Heidel, Andrew J., Hajara M. Lawal, Marius Felder, Christina Schilde, Nicholas R. Helps, Budi Tunggal, Francisco Rivero, et al. 2011. "Phylogeny-Wide Analysis of Social Amoeba Genomes Highlights Ancient Origins for Complex Intercellular Communication." Genome Research 21 (11): 1882-91.

Hirose, Shigenori, Rocio Benabentos, Hsing-I Ho, Adam Kuspa, and Gad Shaulsky. 2011. "Self-

Recognition in Social Amoebae Is Mediated by Allelic Pairs of Tiger Genes." Science 333 (6041): 467-70.

Hudson, Richard Ellis, Juliann Eve Aukema, Claude Rispe, and Denis Roze. 2002. "Altruism,

Cheating, and Anticheater Adaptations in Cellular Slime Molds." The American Naturalist 160 (1): $31-43$.

Jack, Chandra N., Neil Buttery, Boahemaa Adu-Oppong, Michael Powers, Christopher R. L.

Thompson, David C. Queller, and Joan E. Strassmann. 2015. "Migration in the Social Stage of

Dictyostelium Discoideum Amoebae Impacts Competition." PeerJ 3 (October): e1352.

Jack, C. N., B. Adu-Oppong, M. Powers, D. C. Queller, and J. E. Strassmann. 2011. "Cost of

Movement in the Multicellular Stage of the Social Amoebae Dictyostelium Discoideum and D.

Purpureum." Ethology Ecology \& Evolution 23 (4): 358-67.

Kang, Seungho, Alexander K. Tice, Frederick W. Spiegel, Jeffrey D. Silberman, Tomáš Pánek, Ivan Cepicka, Martin Kostka, et al. 2017. "Between a Pod and a Hard Test: The Deep Evolution of Amoebae." Molecular Biology and Evolution 34 (9): 2258-70.

Kaushik, Sonia, and Vidyanand Nanjundiah. 2003. "Evolutionary Questions Raised by Cellular Slime Mould Development." Proc Indian Natl Sci Acad B69: 825-52.

Kokko, Hanna, and Andrés López-Sepulcre. 2006. "From Individual Dispersal to Species Ranges:

Perspectives for a Changing World." Science 313 (5788): 789-91.

Kuzdzal-Fick, Jennie J., Sara A. Fox, Joan E. Strassmann, and David C. Queller. 2011. “High

Relatedness Is Necessary and Sufficient to Maintain Multicellularity in Dictyostelium." Science 334 (6062): 1548-51.

Lahr, Daniel J. G., Laura Wegener Parfrey, Edward A. D. Mitchell, Laura A. Katz, and Enrique Lara. 2011. "The Chastity of Amoebae: Re-Evaluating Evidence for Sex in Amoeboid Organisms." Proceedings. Biological Sciences / The Royal Society 278 (1715): 2081-90. 
Landolt, John C., Steven L. Stephenson, and James C. Cavender. 2006. "Distribution and Ecology of Dictyostelid Cellular Slime Molds in Great Smoky Mountains National Park." Mycologia 98 (4): $541-49$.

Mauriello, Emilia M. F., Tâm Mignot, Zhaomin Yang, and David R. Zusman. 2010. "Gliding Motility Revisited: How Do the Myxobacteria Move without Flagella?" Microbiology and Molecular Biology Reviews: MMBR 74 (2): 229-49.

Mehdiabadi, Natasha J., Chandra N. Jack, Tiffany Talley Farnham, Thomas G. Platt, Sara E. Kalla, Gad Shaulsky, David C. Queller, and Joan E. Strassmann. 2006. "Social Evolution: Kin Preference in a Social Microbe." Nature 442 (7105): 881-82.

Mohri, Kurato, Yu Kiyota, Hidekazu Kuwayama, and Hideko Urushihara. 2013. "Temporal and NonPermanent Division of Labor during Sorocarp Formation in the Social Amoeba Acytostelium Subglobosum." Developmental Biology 375 (2): 202-9.

Nariya, Hirofumi, and Masayori Inouye. 2008. "MazF, an mRNA Interferase, Mediates Programmed Cell Death during Multicellular Myxococcus Development." Cell 132 (1): 55-66.

Nelson, M. K., A. Clark, T. Abe, A. Nomura, N. Yadava, C. J. Funair, K. A. Jermyn, S. Mohanty, R. A. Firtel, and J. G. Williams. 2000. “An F-Box/WD40 Repeat-Containing Protein Important for Dictyostelium Cell-Type Proportioning, Slug Behaviour, and Culmination." Developmental Biology 224 (1): 42-59.

Olive, Lindsay S., and Carmen Stoianovitch. 1960. "Two New Members of the Acrasiales." Bulletin of the Torrey Botanical Club 87 (1): 1-20.

Olive, L. S., and R. L. Blanton. 1980. "Aerial Sorocarp Development by the Aggregative Ciliate, Sorogena Stoianovitchae." The Journal of Protozoology 27 (3): 293-99.

Ostrowski, Elizabeth A. 2019. "Enforcing Cooperation in the Social Amoebae." Current Biology: CB 29 (11): R474-84.

Ostrowski, Elizabeth A., Mariko Katoh, Gad Shaulsky, David C. Queller, and Joan E. Strassmann. 2008. "Kin Discrimination Increases with Genetic Distance in a Social Amoeba." PLoS Biology 6 (11): e287.

Ràfols, I., A. Amagai, Y. Maeda, H. K. MacWilliams, and Y. Sawada. 2001. "Cell Type Proportioning in Dictyostelium Slugs: Lack of Regulation within a 2.5-Fold Tolerance Range." Differentiation; Research in Biological Diversity 67 (4-5): 107-16. 
Raper, Kenneth B. 1940. "Pseudoplasmodium Formation and Organization in Dictyostelium

Discoideum." Journal of the Elisha Mitchell Scientific Society 56 (2): 241-82.

Raper, Kenneth Bryan. 1984. The Dictyostelids. Princeton, NJ: Princeton University Press.

Raper, Kenneth B., Ann C. Worley, and Dietrich Kessler. 1977. "Observations on Guttulinopsis Vulgaris and Guttulinopsis Nivea." Mycologia 69 (5): 1016-30.

Romeralo, Maria, James C. Cavender, John C. Landolt, Steven L. Stephenson, and Sandra L. Baldauf. 2011. "An Expanded Phylogeny of Social Amoebas (Dictyostelia) Shows Increasing Diversity and New Morphological Patterns." BMC Evolutionary Biology 11 (March): 84.

Romeralo, Maria, Anna Skiba, Alejandro Gonzalez-Voyer, Christina Schilde, Hajara Lawal, Sylwia Kedziora, Jim C. Cavender, Gernot Glöckner, Hideko Urushihara, and Pauline Schaap. 2013. "Analysis of Phenotypic Evolution in Dictyostelia Highlights Developmental Plasticity as a Likely Consequence of Colonial Multicellularity." Proceedings. Biological Sciences / The Royal Society 280 (1764): 20130976.

Ruiz-Trillo, Iñaki, Gertraud Burger, Peter W. H. Holland, Nicole King, B. Franz Lang, Andrew J. Roger, and Michael W. Gray. 2007. "The Origins of Multicellularity: A Multi-Taxon Genome Initiative." Trends in Genetics: TIG 23 (3): 113-18.

Schaap, Pauline, Thomas Winckler, Michaela Nelson, Elisa Alvarez-Curto, Barrie Elgie, Hiromitsu Hagiwara, James Cavender, et al. 2006. "Molecular Phylogeny and Evolution of Morphology in the Social Amoebas." Science 314 (5799): 661-63.

Shadwick, Lora L., Frederick W. Spiegel, John D. L. Shadwick, Matthew W. Brown, and Jeffrey D. Silberman. 2009. "Eumycetozoa = Amoebozoa?: SSUrDNA Phylogeny of Protosteloid Slime Molds and Its Significance for the Amoebozoan Supergroup." PloS One 4 (8): e6754.

Smith, Jeff, David C. Queller, and Joan E. Strassmann. 2014. "Fruiting Bodies of the Social Amoeba Dictyostelium Discoideum Increase Spore Transport by Drosophila." BMC Evolutionary Biology 14 (May): 105.

Spiegel, Frederick W., Lora L. Shadwick, George G. Ndiritu, Matthew W. Brown, Maria Aguilar, and John D. Shadwick. 2017. "Protosteloid Amoebae (Protosteliida, Protosporangiida, Cavosteliida, Schizoplasmodiida, Fractoviteliida, and Sporocarpic Members of Vannellida, Centramoebida, and Pellitida)." In Handbook of the Protists, edited by John M. Archibald, Alastair G. B. Simpson, Claudio H. Slamovits, Lynn Margulis, Michael Melkonian, David J. Chapman, and John O. 
Corliss, 1-38. Cham: Springer International Publishing.

Stephenson, Steven L., and John C. Landolt. 1992. "Vertebrates as Vectors of Cellular Slime Moulds in Temperate Forests." Mycological Research 96 (8): 670-72.

Stephenson, Steven L., and Martin Schnittler. 2017. "Myxomycetes." In Handbook of the Protists, edited by John M. Archibald, Alastair G. B. Simpson, Claudio H. Slamovits, Lynn Margulis, Michael Melkonian, David J. Chapman, and John O. Corliss, 1-27. Cham: Springer International Publishing.

Sucgang, Richard, Alan Kuo, Xiangjun Tian, William Salerno, Anup Parikh, Christa L. Feasley, Eileen Dalin, et al. 2011. "Comparative Genomics of the Social Amoebae Dictyostelium Discoideum and Dictyostelium Purpureum." Genome Biology 12 (2): R20.

Sugimoto, Hiroki, and Hiroshi Endoh. 2006. "Analysis of Fruiting Body Development in the Aggregative Ciliate Sorogena Stoianovitchae (Ciliophora, Colpodea)." The Journal of Eukaryotic Microbiology 53 (2): 96-102.

Swanson, Andrew R., Eduardo M. Vadell, and James C. Cavender. 1999. "Global Distribution of Forest Soil Dictyostelids." Journal of Biogeography 26 (1): 133-48.

Tarnita, Corina E., Clifford H. Taubes, and Martin A. Nowak. 2013. "Evolutionary Construction by Staying Together and Coming Together." Journal of Theoretical Biology 320 (March): 10-22.

Tice, Alexander K., Jeffrey D. Silberman, Austin C. Walthall, Khoa N. D. Le, Frederick W. Spiegel, and Matthew W. Brown. 2016. "Sorodiplophrys Stercorea: Another Novel Lineage of Sorocarpic Multicellularity." The Journal of Eukaryotic Microbiology 63 (5): 623-28.

Varon, Mazal, Shulamit Cohen, and Eugene Rosenberg. 1984. "Autocides Produced by Myxococcus Xanthus." Journal of Bacteriology 160 (3): 1146-50.

Velicer, Gregory J., and Michiel Vos. 2009. "Sociobiology of the Myxobacteria." Annual Review of Microbiology 63: 599-623.

Votaw, H. R., and E. A. Ostrowski. 2017. "Stalk Size and Altruism Investment within and among Populations of the Social Amoeba." Journal of Evolutionary Biology 30 (11): 2017-30. Williams, Jeffrey G. 2010. "Dictyostelium Finds New Roles to Model.” Genetics 185 (3): 717-26. Worley, Ann C., Kenneth B. Raper, and Marianne Hohl. 1979. "Fonticula Alba: A New Cellular Slime Mold (Acrasiomycetes)." Mycologia 71 (4): 746-60. 